The Journal of Laryngology \& Otology

http://journals.cambridge.org/JLO

Additional services for The Journal of Laryngology \& Otology:

Email alerts: $\underline{\text { Click here }}$

Subscriptions: $\underline{\text { Click here }}$

Commercial reprints: $\underline{\text { Click here }}$

Terms of use : $\underline{\text { Click here }}$

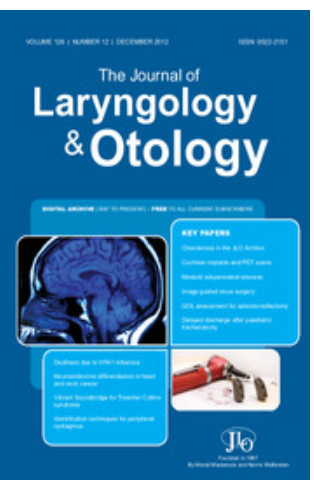

\title{
Comparison of techniques for identification of peripheral vestibular nystagmus
}

P D B West, Z A Sheppard and E V King

The Journal of Laryngology \& Otology / Volume 126 / Issue 12 / December 2012, pp 1209 - 1215

DOI: 10.1017/S0022215112002368, Published online:

Link to this article: http://journals.cambridge.org/abstract_S0022215112002368

How to cite this article:

P D B West, Z A Sheppard and E V King (2012). Comparison of techniques for identification of peripheral vestibular nystagmus. The Journal of Laryngology \& Otology, 126, pp 1209-1215 doi:10.1017/S0022215112002368

Request Permissions : $\underline{\text { Click here }}$ 


\title{
Comparison of techniques for identification of peripheral vestibular nystagmus
}

\author{
P D B WEST ${ }^{1}$, Z A SHEPPARD ${ }^{2}, \mathrm{E} \mathrm{V} \mathrm{KING}^{3,4}$ \\ ${ }^{1}$ Department of Audiovestibular Medicine, Queen Alexandra Hospital, Portsmouth, ${ }^{2}$ School of Health and Social \\ Care, Bournemouth University, ${ }^{3}$ Department of Otorhinolaryngology, Head and Neck Surgery, Poole Hospital \\ NHS Foundation Trust, and ${ }^{4}$ Cancer Sciences Division, University of Southampton, UK
}

\begin{abstract}
Objective: To determine the best clinical method for identifying peripheral vestibular nystagmus, by comparing eye movement examination with optic fixation, and with fixation removed using Frenzel's glasses, infra-red videoFrenzel's goggles or an ophthalmoscope, with results of electronystagmography.

Method: One hundred patients referred for electronystagmography from the audiovestibular medicine clinic at Queen Alexandra Hospital, Portsmouth, were examined immediately before undergoing electronystagmography.

Results: Video-Frenzel's goggles were highly effective at detecting peripheral vestibular nystagmus, with a sensitivity of 85 per cent ( 95 per cent confidence interval, 62.1-96.8 per cent) and a specificity of 65 per cent (53.5-75.3 per cent), compared with electronystagmography. Ophthalmoscopy had comparable sensitivity to Frenzel's glasses (used in the dark), i.e. 26.3 per cent (9.1-51.2 per cent) compared with 31.6 per cent (12.6-56.6 per cent), respectively. Frenzel's glasses as normally used in ENT clinics (i.e. in dim lighting) were ineffective, with a sensitivity of just 10 per cent (1.2-31.7 per cent).

Conclusion: Video-Frenzel's goggles should be used in all clinics with substantial numbers of balance-impaired patients. Traditional Frenzel's glasses have no place in clinical practice unless formal black-out facilities are available.
\end{abstract}

Key words: Vertigo; Diagnosis; Nystagmus; Electronystagmography; Frenzel's Glasses

\section{Introduction}

One of the cardinal diagnostic features of vestibular disorders is the presence of jerk nystagmus: an involuntary, rhythmic, to and fro movement of the eyes, with alternating slow drift (the pathological phase) and rapid, corrective saccades. In clinical practice, it is vital to be able to distinguish between nystagmus of peripheral origin (due to inner ear or vestibular nerve pathology) versus central origin (due to brainstem or cerebellar pathology). One of the crucial distinguishing features is that horizontal nystagmus of inner-ear origin is suppressed by visual fixation, whereas central nystagmus is either unaffected or may even be enhanced by fixation. Indeed, so effective is the fixation mechanism at overriding peripheral vestibular nystagmus that such nystagmus will generally only be apparent on routine examination during the most acute phase of a vestibular disorder. As a result, many patients with peripheral vestibular imbalance remain undiagnosed because no abnormality is found on examination.
Therefore, in order to diagnose vestibular disorders with any degree of certainty, the patient must be examined in the absence of optic fixation. Where this is done in ENT clinics, Frenzel's glasses (Figure 1) have traditionally been used. These consist of 20 diopter illuminated lenses and should be used in total darkness. ${ }^{1}$ In practice, as few out-patient clinic rooms have a black-out facility, they are generally used in dim light, and are still assumed to prevent the patient from fixating. Because the illumination is provided at the sides and the patient is therefore asked to look straight ahead, the examiner is prevented from taking advantage of Alexander's law, which states that nystagmus is enhanced when the subject looks in the direction of the fast phase. ${ }^{2}$

Another way of preventing visual fixation is by ophthalmoscopic examination of the optic fundus, with the other eye covered. (An ophthalmoscope with a bright halogen light source must be used in order to prevent fixation on the light; this will additionally prevent possible contamination of results by the 


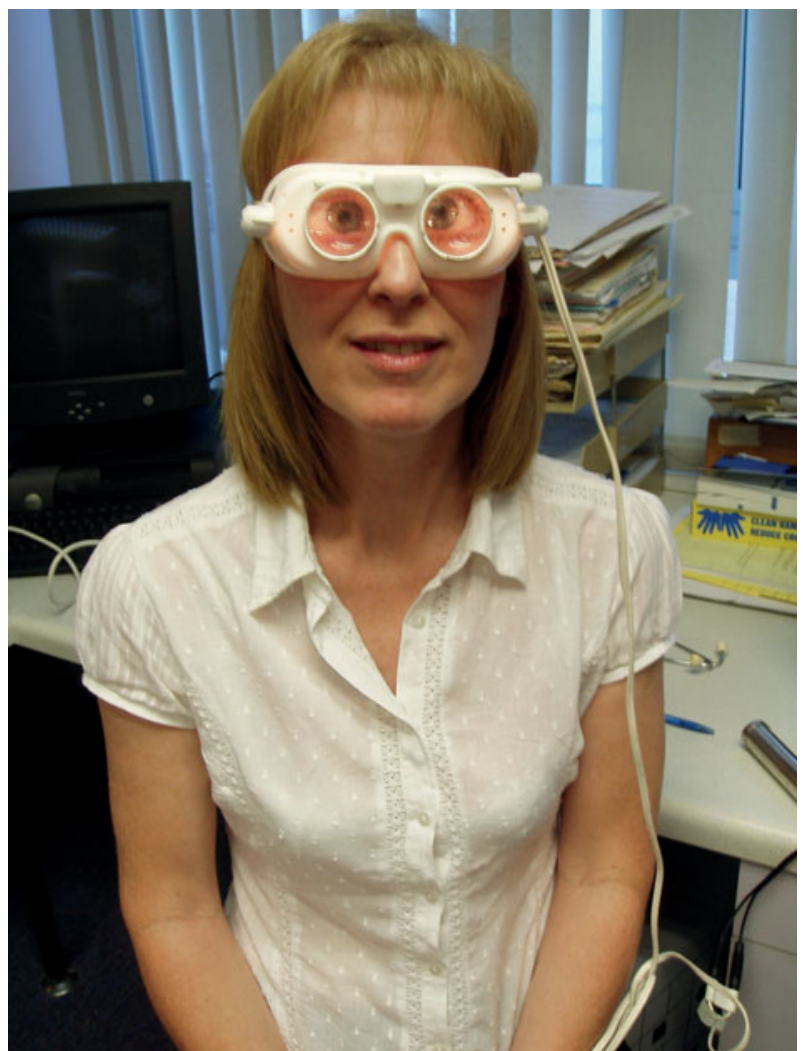

FIG. 1

Frenzel's glasses in use in a dimly lit room. Published with subject's permission.

emergence of latent nystagmus, a variant of congenital nystagmus mainly encountered in persons with strabismus and amblyopia, which might otherwise be present when one eye is covered.) This examination could readily be performed in general practice or in hospital accident and emergency departments, where most cases of acute vertigo are seen.

In specialist hospital balance clinics, nystagmus is generally observed and recorded using electronystagmography (ENG) or, increasingly, computerised infra-red

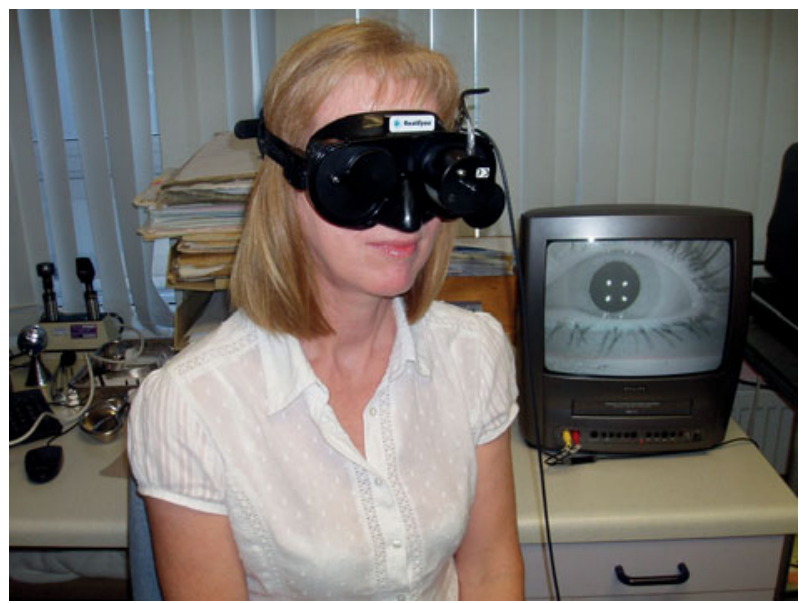

FIG. 2

Video-Frenzel's goggles in use, showing video display. video-nystagmography. These techniques are considered by many to be the 'gold standard' as results are both objective and quantifiable, in terms of the slow-phase velocity of any nystagmus. However, these investigations are specialised, time-consuming and (therefore) expensive, and are not available in general practice or in smaller ENT clinics or audiology departments.

In recent years, the investigation of nystagmus has been revolutionised by the development and ready availability (at modest cost) of infra-red videoFrenzel's goggles which connect either to a video cassette recorder and television (Figure 2) or a computer. All patients attending the first author's audiovestibular medicine clinics are routinely examined using these video-Frenzel's goggles. Thus, most peripheral vestibular disorders are diagnosed (and distinguished from central disorders) without the need for referral for ENG or video-nystagmography.

This change in practice prompts the following questions: (1) are cases being under- or over-diagnosed because ENG is not commonly being undertaken; (2) how effective are video-Frenzel's goggles compared with ENG, and how much better are they than the vastly cheaper Frenzel's glasses (which are still used in many ENT clinics); and (3) could the ophthalmoscope provide a viable alternative in general practice?

Few published papers have directly compared different methods of nystagmus examination.

Ben-David et al. $^{3}$ and Strauss and Meyer zum Gottesberge $^{4}$ found no difference between ENG and Frenzel's glasses.

More recently, Baba et al. $^{5}$ examined 100 vertiginous patients using ENG, with fixation abolished either by Frenzel's glasses or with an infra-red camera. All 100 patients showed nystagmus with the infra-red camera, which suggests that those who did not show nystagmus were excluded from the study; the paper is ambiguous on this point. Only 33 patients showed nystagmus under Frenzel's glasses, and in those cases the slow-phase velocity was significantly lower ( $p<0.001$; paired $t$-test) than with the camera. Whilst the authors concluded that the infra-red camera was 'three times more sensitive' than Frenzel's glasses and should be used more widely, the 100 per cent detection rate raises the possibility that the camera might be over-sensitive, detecting nystagmus that was possibly physiological.

A similar concern had previously been expressed by Mulch and Lewitzki, ${ }^{6}$ who recommended that ENG be carried out with Frenzel's glasses rather than with eyes open in the dark.

Whilst Guidetti et al. ${ }^{7}$ strongly promoted the routine use of video-Frenzel's goggles, their study was not directly comparable with the current one as they did not assess the detection of nystagmus per se, but rather examined the identification of peripheral vestibular hypofunction, using bithermal caloric testing as the gold standard. Examining for positioning and head-shaking as well as for spontaneous nystagmus, 
they reported that video-Frenzel's goggles enabled a peripheral vestibular defect to be identified correctly in over 77 per cent of cases, compared with approximately 50 per cent when eyes were examined with fixation or with Frenzel's glasses. This difference was sufficient for the authors to recommend that videoFrenzel's goggles be used to screen for a labyrinthine defect in patients with vertigo.

No previous studies have included ophthalmoscopic fundoscopy, which, if effective at identifying nystagmus, would enable easier diagnosis in non-specialist settings, without the need for expensive equipment. The present research was designed to compare the effectiveness of the clinical examination techniques routinely available for the detection and evaluation of nystagmus in the out-patient clinic (i.e. the ophthalmoscope, Frenzel's glasses (in both dim and dark conditions) and video-Frenzel's goggles), in terms of their relative sensitivity, specificity and predictive value, especially in relation to ENG.

\section{Materials and methods}

\section{Ethical consideration and patient recruitment}

Ethical approval was obtained from the Isle of Wight, Portsmouth and South East Hampshire Research Ethics Committee.

One hundred and ten patients (over the age of 18 years) were recruited from those who attended the first author's audiovestibular medicine clinic between February 2005 and April 2009 for evaluation of dizziness and imbalance, and who were referred on to the audiology department for ENG and caloric testing. All new patients attending the audiovestibular medicine clinic underwent a full clinical neuro-otological examination, including the use of video-Frenzel's goggles to detect the presence of spontaneous, gaze-evoked and head-shaking nystagmus. Only approximately 5 per cent of new patients were additionally referred for ENG and caloric testing. Such testing was requested for the following principal reasons: (1) to identify the characteristics and to quantify the slow-phase velocity of nystagmus found on clinical examination, in order to determine its possible significance; (2) to confirm and quantify the extent of any peripheral vestibular impairment; (3) to confirm the presence, extent and nature of vestibular impairment in patients who had failed to respond to treatment; (4) to determine whether unilateral hearing loss was accompanied by unilateral vestibular impairment; and (5) to confirm a diagnosis of bilateral vestibular failure and to quantify its extent.

Thus, patients referred for ENG and caloric testing and recruited for the study were not selected specifically on the grounds of having (or not having) nystagmus on examination.

Ten patients who had originally given their consent did not participate, either due to cancellation or failure to attend their ENG appointment. In most cases, this was because their symptoms had resolved.

Patients were given both verbal and written information about the study.

\section{Exclusion criteria}

Patients with suspected central vestibular disorders, on the grounds of either history or examination, were excluded. This criterion included any patient in whom vertical nystagmus was detected. Therefore, all recorded nystagmus was horizontal. Patients whose eye movements were difficult to assess were also excluded; in two cases, claustrophobia prevented the use of light-proof goggles.

\section{Patient examination}

Patients attending for ENG were asked to arrive 10 minutes early, in order to be re-examined by the first author personally, immediately prior to their ENG.

Patients were examined: (1) for spontaneous and gaze-evoked nystagmus using optic fixation; (2) for spontaneous nystagmus with fixation removed using the ophthalmoscope, Frenzel's glasses in dim light and Frenzel's glasses in the dark; and (3) for spontaneous and gaze-evoked nystagmus with fixation removed using video-Frenzel's goggles. Whilst the order of performance of these examinations was kept constant, the starting point was rotated in order to minimise observer bias. For each examination (carried out for a minimum of 10 seconds), the presence or absence of horizontal nystagmus was recorded, together with its direction and, where appropriate, its degree according to Alexander's law. ${ }^{2}$ As the nystagmus revealed by these examinations was not quantifiable, no attempt was made to estimate slow-phase velocity. The presence of any persistent nystagmus was noted. (Fine, symmetrical, end-point nystagmus was frequently observed on lateral gaze using videoFrenzel's goggles, as the angle of gaze could easily exceed the $30^{\circ}$ used in the ENG examination. In this study, first degree nystagmus on lateral gaze was deemed to be present only if it was clearly asymmetrical, i.e. only present on gaze to one side.)

Results were then compared with the subsequent ENG result for each test condition on an individual patient basis. In order for significant nystagmus to be deemed to be present on ENG, the criterion officially adopted by the audiology department specified a slow-phase velocity of $4^{\circ}$ per second or greater. ${ }^{8}$ In practice, the audiologists reporting ENG traces used a degree of discretion, and nystagmus was reported as being present with slow-phase velocities as low as $2^{\circ}$ per second, as long as it was consistent, of (at least) second degree, and did not disobey Alexander's law.

The fact that all the tests were performed in one session minimised any temporal variation in the presence or magnitude of nystagmus. As all clinical examinations with a subjective component were performed by one physician, inter-observer error was eliminated. 
Towards the end of the trial, ENG began to be replaced by video-nystagmography. Therefore, the final three patients underwent video-nystagmography instead of ENG. In these cases, the video-nystagmography results were included with the 'ENG eyes open in dark' results.

\section{Caloric testing}

All patients undergoing ENG examination also underwent bithermal caloric testing (in accordance with the 'Recommended Procedure' published by the British Society of Audiology) $;{ }^{9}$ indeed, it was for this reason that most, if not all, of the patients included in this study had been referred for investigation. For this reason, and also in the light of Guidetti and colleagues' results $^{7}$ (see above), it was decided also to determine whether there was an association between the presence or absence of nystagmus and the presence or absence of a clinically significant canal paresis (derived by Jongkees' formula $)^{10}$ of 20 per cent or greater, defined according to normative data.

\section{Statistical analysis}

The accuracy of each clinical examination technique was compared with ENG (adopted as the reference standard, because results were objective and quantifiable), using the following four different statistical measures: ${ }^{11}$ (1) sensitivity (i.e. the percentage of patients with nystagmus on ENG who also tested positively for nystagmus using the method in question); (2) specificity (i.e. the percentage without nystagmus on ENG who tested negatively for nystagmus using the method in question); (3) positive predictive value (i.e. the percentage who tested positively for nystagmus using the method in question, who had nystagmus on ENG); and (4) negative predictive value (i.e. the percentage who tested negatively for nystagmus using the method in question, who did not have nystagmus on ENG).

These four measures were calculated using the Statistical Package for the Social Sciences program and its updated version, the Predictive Analytics Software program (IBM, Armonk, New York, USA).

For a diagnostic test to be considered clinically useful, a high sensitivity, specificity, positive predictive value and negative predictive value are all desirable. However, the relative importance of each of these statistical measures in any particular clinical scenario is debatable. The authors' view is that, in the diagnosis of peripheral vestibular disorders, sensitivity is the most important measure, in order to ensure that patients who have nystagmus are not missed, even at the expense of detecting apparent nystagmus which eventually is found to be of no clinical significance. This issue is discussed in more detail below. However, both the positive predictive value and the negative predictive value will depend on the prevalence of nystagmus. ${ }^{11}$ All test results were classified as either positive or negative (i.e. nystagmus judged to be either present or absent). The precision of estimates of the statistical measures was summarised with 95 per cent confidence intervals (CIs), using the exact method recommended by Bland ${ }^{11}$ and using his Biconf software. ${ }^{11}$ The possibility of an association between the presence or absence of nystagmus on each test and the presence or absence of a canal paresis on caloric testing was investigated using the chi-square test (or, where appropriate (in one case), Fisher's exact test).

\section{Results}

Of the 100 patients who completed the study, 51 were female and 49 male, with a mean age of 50.9 years (standard deviation $=13.6$ years). Table I shows the number of patients in whom (horizontal) nystagmus was detected by each test in turn. Electronystagmography results were reported with eyes open in the light (i.e. with fixation) and with eyes open in the dark (i.e. with fixation removed). Electronystagmography results were labelled as positive if nystagmus was detected. In no case was nystagmus present with fixation. Twenty patients had detectable nystagmus on ENG with fixation removed. More than twice as many $(n=45)$ had nystagmus with video-Frenzel's goggles, as opposed to just 10 with Frenzel's glasses in the dark and only 3 with Frenzel's glasses in dim lighting.

The sensitivity, specificity, positive predictive value and negative predictive value of the various examination techniques, in comparison with ENG, are presented in Table II. Video-Frenzel's goggles had the highest sensitivity (85 per cent $(95$ per cent CI, 62.1-96.8 per cent)) and negative predictive value (94.5 per cent (84.9-98.9 per cent)), with a moderate specificity (65 per cent (53.5-75.3 per cent), lower than that of Frenzel's glasses) but a low positive predictive value (37.8 per cent $(23.8-53.5$ per cent)). Frenzel's glasses in the dark were only marginally more sensitive (31.6 per cent (12.6-56.6 per cent)) than the ophthalmoscope (26.3 per cent $(9.1-51.2$ per cent)), with a very similar negative predictive value, but greater specificity $(95.0$ per cent (87.7-98.6 per cent) against 77.5 per cent $(66.8-86.1$ per cent) for the ophthalmoscope) and, especially, greater positive predictive value $(60.0$ per cent

\begin{tabular}{lrrr}
\multicolumn{4}{c}{ TABLE I } \\
\multicolumn{2}{c}{ NYSTAGMUS DETECTION BY EACH TEST* } \\
\hline \multirow{2}{*}{ Test } & \multicolumn{2}{c}{ Nystagmus } & \multirow{2}{*}{ Missing data } \\
\cline { 2 - 3 } & Present & Absent & \\
\hline Ophthalmoscope & 23 & 76 & 1 \\
Frenzel's glasses (dim light) & 3 & 96 & 1 \\
Frenzel's glasses (dark room) & 10 & 89 & 1 \\
Video-Frenzel's goggles & 45 & 55 & 0 \\
ENG with fixation & 0 & 100 & 0 \\
ENG with eyes open in dark & 20 & 80 & 0 \\
\hline
\end{tabular}

Data represent patient numbers (and also percentages, as $n=100$ ). * In 100 patients attending the audiovestibular medicine clinic February 2005 to April 2009. ENG = electronystagmography 
TABLE II

STATISTICAL MEASURES OF TESTS*, COMPARED WITH ELECTRONYSTAGMOGRAPHY ${ }^{\dagger}$

\begin{tabular}{lllll}
\hline Test & \multicolumn{1}{c}{ Sensitivity } & Specificity & PPV & NPV \\
\hline Ophthalmoscope $^{\ddagger}$ & $26.3(9.1-51.2)$ & $77.5(66.8-86.1)$ & $21.7(7.5-43.7)$ & $81.6(71.0-89.5)$ \\
Frenzel's glasses (dim light) $^{\ddagger}$ & $10.0(1.2-31.7)$ & $98.7(93.1-100.0)$ & $66.7(9.4-99.2)$ & $81.3(72.0-88.5)$ \\
Frenzel's glasses (dark room) $^{\ddagger}$ & $31.6(12.6-56.6)$ & $95.0(87.7-98.6)$ & $60.0(26.2-87.8)$ & $85.4(76.3-92.0)$ \\
Video-Frenzel's goggles** $^{*}$ & $85.0(62.1-96.8)$ & $65.0(53.5-75.3)$ & $37.8(23.8-53.5)$ & $94.5(84.9-98.9)$ \\
\hline
\end{tabular}

Data represent percentages (95 per cent confidence intervals, exact method). *For patients attending the audiovestibular medicine clinic between February 2005 and April 2009. ${ }^{\dagger}$ Performed with eyes open in the dark. ${ }^{*} n=99 ;{ }^{* *} n=100$. PPV $=$ positive predictive value; $\mathrm{NPV}=$ negative predictive value

(26.2-87.8 per cent) versus 21.7 per cent $(7.5-43.7)$ for the ophthalmoscope).

In this study, video-Frenzel's goggles detected more than twice as many cases of nystagmus as ENG with eyes open in the dark: 45 compared with 20 . The question therefore arises as to whether video-Frenzel's goggles were detecting false positives (i.e. nystagmus that was not clinically significant) or whether, conversely, ENG might be failing to detect a proportion of genuine cases of clinically significant nystagmus. The possible effect on this issue of the slow-phase velocity criterion used in reporting ENG results is discussed below.

An alternative way of helping to answer this question was to re-analyse the data using video-Frenzel's goggles as the reference point (Table III). The sensitivity of ENG when compared to video-Frenzel's goggles was just 37.8 per cent (95 per cent CI, 23.8-53.5 per cent), although ENG had a high specificity (94.5 per cent (84.9-98.9)) and positive predictive value ( 85 per cent $(62.1-96.8$ per cent)). The other main effect of this analysis was to increase the sensitivity of ophthalmoscopic fundoscopy to 34.1 per cent (20.5-49.9 per cent), with a specificity of 85.5 per cent (73.3-93.5 per cent). This compares favourably with Frenzel's glasses which, even in the dark, had a sensitivity of just 20.5 per cent $(9.8-35.3$ per cent).

Ninety-six patients completed full bithermal caloric testing, of whom 31 had clinically significant canal paresis. The relationship between the presence or absence of nystagmus on each test and the presence or absence of a canal paresis is shown in Table IV. Although multiple significance tests were conducted, only in the case of nystagmus detected by ENG was there a significant association ( $p=0.003$, chi-square test) between the presence of nystagmus and the presence of a canal paresis.

\section{Discussion}

This study was designed to determine the most appropriate clinical examination method for demonstrating nystagmus due to peripheral vestibular disorders. Such nystagmus may present in a variety of clinical settings: not only in audiovestibular medicine and ENT clinics, but also in general practice, general medicine, neurology and neurosurgery.

It will be noted that it took four years to recruit 100 patients to this study. The routine use of video-Frenzel examination in the first author's clinic has enabled diagnosis to be made in a high proportion of cases, with fewer than 5 per cent of new patients presenting with balance disorders needing to be referred for ENG or video-nystagmography and caloric testing.

The study showed that in no case was peripheral vestibular nystagmus observed in the presence of optic fixation: the only clinical examination carried out by most medical practitioners. Such nystagmus, if present, would have been indicative of either an acute peripheral vestibular event (in which case it would be enhanced by removing fixation, and the patient would probably be acutely vertiginous) or a central vestibular disorder. As long ago as 1980, Reker $^{1}$ noted that weak peripheral vestibular nystagmus could only be recognised using Frenzel's glasses following a 'long and careful search in a totally dark room'. The present results confirmed that, in dim light (as they are generally used in ENT clinics), Frenzel's glasses were of little value: their sensitivity compared with ENG was only 10.0 per cent $(95$ per cent CI, 1.2-31.7 per cent), and their sensitivity

\begin{tabular}{lcccc}
\multicolumn{5}{c}{ TABLE III } \\
\hline Test & \multicolumn{5}{c}{ Sensitivity } & Specificity & PPV & NPV \\
\hline Ophthalmoscope $^{\dagger}$ & $34.1(20.5-49.9)$ & $85.5(73.3-93.5)$ & $65.2(42.7-83.6)$ & $61.8(50.0-72.8)$ \\
Frenzel's glasses (dim light) $^{\dagger}$ & $6.7(1.4-18.3)$ & $100.0(93.4-100.0)$ & $100.0(29.2-100.0)$ & $56.3(45.7-66.4)$ \\
Frenzel's glasses (dark room) $^{\dagger}$ & $20.5(9.8-35.3)$ & $98.2(90.3-100.0)$ & $90.0(55.5-99.7)$ & $60.7(49.8-70.9)$ \\
ENG (eyes open in dark) $^{\ddagger}$ & $37.8(23.8-53.5)$ & $94.5(84.9-98.9)$ & $85.0(62.1-96.8)$ & $65.0(53.5-75.3)$ \\
\hline
\end{tabular}

Data represent percentages $\left(95\right.$ per cent confidence intervals, exact method). ${ }^{*}$ For patients attending the audiovestibular medicine clinic between February 2005 and April $2009 .{ }^{\dagger} n=99 ;{ }^{\star} n=100$. PPV = positive predictive value; NPV = negative predictive value; $\mathrm{ENG}=$ electronystagmography 
TABLE IV

RELATIONSHIP BETWEEN CANAL PARESIS BY CALORIC TESTING AND NYSTAGMUS BY EACH DIAGNOSTIC TEST*

\begin{tabular}{|c|c|c|c|c|c|c|c|c|c|c|c|c|}
\hline \multirow[t]{2}{*}{$\mathrm{CP}^{\dagger}$} & \multicolumn{3}{|c|}{ Nyst by Ophth ${ }^{\ddagger}$} & \multicolumn{3}{|c|}{ Nyst by FG (in dark) } & \multicolumn{3}{|c|}{ Nyst by video-F** } & \multicolumn{3}{|c|}{ Nyst by ENG (in dark)** } \\
\hline & No & Yes & $p$ & No & Yes & $p$ & No & Yes & $p$ & No & Yes & $p$ \\
\hline No & 54 & 11 & $0.073^{\S}$ & 61 & 4 & $0.135^{\#}$ & 39 & 26 & $0.172^{\S}$ & 57 & 8 & $0.003^{\S}$ \\
\hline Yes & 20 & 10 & & 25 & 5 & & 14 & 17 & & 19 & 12 & \\
\hline
\end{tabular}

*In 100 patients attending the audiovestibular medicine clinic between February 2005 and April 2009 in whom caloric testing was successfully completed. ${ }^{\dagger}$ Canal paresis (CP) of 20 per cent or more on caloric testing. ${ }^{\star} n=95 ;{ }^{* *} n=96 .{ }^{\S}$ Chi-square test; ${ }^{\sharp}$ Fisher's exact test. Nyst $=$ nystagmus; Ophth = ophthalmoscopy; FG = Frenzel's glasses; video-F = video-Frenzel's goggles; ENG = electronystagmography (eyes open in a dark room); No = absent; Yes = present

compared with video-Frenzel's goggles (see below) just 6.7 per cent (1.4-18.3 per cent). Even in darkness, their sensitivity was only comparable to that of the ophthalmoscope. However, despite having poor sensitivity, both the ophthalmoscope and Frenzel's glasses (in darkness) had reasonable specificity.

Concern has previously been expressed that ENG might be too sensitive, demonstrating nystagmus that is essentially physiological. Mulch and Lewitzki ${ }^{6}$ found evidence of horizontal nystagmus using ENG with eyes open in the dark in 63 out of 102 apparently normal subjects. In only two of these cases was nystagmus visible using Frenzel's glasses in a dark room: an even smaller proportion than that found in the present study. Similarly, Coates ${ }^{12}$ found spontaneous nystagmus using ENG with eyes closed in 20-25 per cent of both normal $(n=121)$ and patient $(n=1601)$ groups, and suggested that only spontaneous nystagmus with a slow-phase velocity of $10^{\circ}$ per second or greater could be considered definitely pathological. Nowadays, a figure of $4^{\circ}$ per second is widely accepted. ${ }^{8}$

In the present research, video-Frenzel's goggles clearly constituted the most sensitive method for detecting nystagmus, but at the expense of possibly reduced specificity and positive predictive value, certainly in comparison with ENG. There is no doubt that the 'objective' methods (ENG and video-nystagmography) can fail to record nystagmus that is clearly present. The first author has seen cases where this has occurred, due either to artefactual electrical noise (in the case of ENG), or to failure of the video-nystagmography system to focus on the subject's pupil due to long eyelashes or eye make-up. However, the low sensitivity of ENG when compared with video-Frenzel's goggles cannot be explained purely in terms of technical failure, and there remains the key question of which method is better at detecting 'genuine' cases.

One possibility is that genuine cases of nystagmus detected by ENG were discounted by setting an essentially arbitrary slow-phase velocity criterion. As already stated, the widely accepted figure of $4^{\circ}$ per second ${ }^{8}$ is historical and may be unduly strict, especially given the recent advent of computerised videonystagmograhy, which results in less 'noisy' traces. It may be appropriate for audiologists to adopt a less stringent criterion, as in this study. Of the 20 cases with nystagmus reported as being present on ENG, only 5 actually had slow-phase velocities of $4^{\circ}$ per second or greater (nystagmus was present using video-Frenzel's goggles in all of these). In 15 of the 20 cases, although the slow-phase velocities were just 2 or $3^{\circ}$ per second, the nystagmus, of second or third degree, was consistent and obeyed Alexander's law. In an additional 12 cases, purely first degree nystagmus with a slow-phase velocity of 2 or $3^{\circ}$ per second was discounted by the audiologist reporting the ENG as likely to be artefactual, either because it was inconsistent (or difficult to discern because of excessively noisy traces) or disobeyed Alexander's law. In these cases, nystagmus was reported by the audiologist as 'absent'. In only 4 of these 12 cases was nystagmus apparent on examination with video-Frenzel's goggles. In an additional four cases, the ENG traces were too noisy for any subtle nystagmus to be identifiable. In none of these four was nystagmus apparent on examination with video-Frenzel's goggles. Thus, the slow-phase velocity criterion cannot explain the difference in the nystagmus detection rates of video-Frenzel's goggles and ENG.

Another possible way of assessing whether videoFrenzel examination was potentially over-sensitive was to assess the relationship between the presence of nystagmus detected by each test in turn and the presence of unilateral vestibular hypofunction as indicated by the presence of a canal paresis on bithermal caloric testing (following Guidetti et al.). ${ }^{7}$ Although multiple significance tests were conducted, only in the case of nystagmus detected by ENG was there a statistically significant association between the presence or absence of nystagmus and the presence or absence of a canal paresis. This might suggest that $\mathrm{ENG}$, in recording nystagmus with a quantifiable slow-phase velocity, is the most robust measure. However, the detection of nystagmus and the detection of canal paresis on caloric testing represent entirely different parameters of vestibular function. Even given the severe limitations of caloric testing (an unphysiological stimulus which only measures ultralow frequency vestibular function), a canal paresis implies impairment of horizontal semi-circular canal function, whereas the presence or absence of nystagmus is a dynamic sign relating to differences in tonic vestibular activity on the two sides (dependent also on central compensatory mechanisms) which may be independent of such impairment. Thus, a patient 
recovering from an acute Ménière's attack may have nystagmus but no canal paresis. Conversely, a patient with total unilateral vestibular failure will, in the acute phase, be severely vertiginous and will have profound nystagmus. As central compensation occurs, even in the absence of recovery of peripheral function, nystagmus will diminish along with symptoms. As the nystagmus diminishes, there will come a point at which the slow-phase velocity will drop below any (necessarily arbitrary) cut-off point, whilst the patient may remain symptomatic. Thus, the presence and magnitude of nystagmus may correlate with symptoms far better than do the results of caloric testing.

- Peripheral vestibular nystagmus can be difficult to diagnose in the clinic

- Video-Frenzel's goggles should be used routinely in all clinics with substantial numbers of balance-impaired patients

- Traditional Frenzel's glasses should not be used without formal black-out facilities

- Fundoscopic examination with an ophthalmoscope is a possible alternative to video-Frenzel's goggles, for screening

Inevitably, there is a trade off between sensitivity, specificity, positive predictive value and negative predictive value. In the identification of peripheral vestibular disorders, sensitivity may be considered of greater importance than specificity, as the key consideration is that patients with possible peripheral vestibular imbalance should not be missed. In cases of possible false positives, the onus is on the clinician to interpret physical signs in the light of the whole clinical picture, in order to judge their potential significance in any given case. Furthermore, the consequences of treating (usually with vestibular rehabilitation exercises) an erroneously diagnosed peripheral vestibular imbalance are arguably less severe for the patient than those of failing to diagnose a potentially treatable disorder. The high sensitivity of video-Frenzel's goggles in detecting nystagmus should be balanced against their relatively low specificity and positive predictive value (when compared with ENG) in enabling the clinician to come to a fully informed judgment as to the probable origin of any particular patient's symptoms of dizziness or imbalance.

\section{Conclusion}

These study findings support the authors' view that video-Frenzel's goggles provide a simple, quick and relatively inexpensive method of detecting peripheral vestibular nystagmus, and constitute an invaluable diagnostic tool with which every vestibular clinic ought now to be equipped.

The study also provides some support for the use of ophthalmoscopic fundoscopy in the evaluation of the dizzy patient in less specialised settings, without the need for recourse to specialist referral and investigation. In the UK, the Department of Health's recently published balance 'Good Practice Guide" ${ }^{13}$ stressed the need for better diagnosis and management of these patients in the primary care setting. The instruction of primary care physicians in the use of fundoscopy in the diagnosis of vestibular disorders might be a good first step.

\section{Acknowledgements}

We are grateful for the help of the audiological team (especially Andy Rutter) at Portsmouth Hospitals NHS Trust; for permission from the Chief Audiologist, Jane Hotchkiss, to publish unflattering photographs; and for the statistical advice provided by Peter Thomas of Bournemouth University.

References

1 Reker U. Peripheral-vestibular spontaneous nystagmus. Analysis of reproducibility and methodologies. Arch Otorhinolaryngol 1980;226:225-237

2 Jeffcoat B, Shelukhin A, Fong A, Mustain W, Zhou W. Alexander's Law revisited. J Neurophysiol 2008;100:154-9

3 Ben-David Y, Hafner H, Fradis M, Krasnitz G, Podoshin L. Do Frenzel glasses have a place in the modern electronystagmography laboratory? Am J Otol 1996;17:89-92

4 Strauss P, Meyer zum Gottesberge A. Caloric nystagmus: ENG in comparison with observation by Frenzel's glasses. Adv Otorhinolaryngol 1979;25:134-7

5 Baba S, Fukumoto A, Aoyagi M, Koizumi Y, Ikezono T, Yagi T. A comparative study on the observation of spontaneous nystagmus with Frenzel glasses and an infrared CCD camera. Journal of the Nippon Medical School 2004;71:25-9

6 Mulch G, Lewitzki W. Spontaneous and positional nystagmus in healthy persons demonstrated only by electronystagmography: physiological spontaneous nystagmus or "functional scar"? Arch Otorhinolaryngol 1977;215:135-45

7 Guidetti G, Monzani D, Rovatti V. Clinical examination of labyrinthine-defective patients out of the vertigo attack: sensitivity and specificity of three low-cost methods. Acta Otorhinolaryngol Ital 2006;26:96-101

8 Sills AW, Baloh RW, Honrubia V. Caloric testing 2. Results in normal subjects. Ann Otol Rhinol Laryngol Suppl 1977; 86(suppl 43):7-23

9 British Society of Audiology: Recommended procedure: The caloric test. In: http://www.thebsa.org.uk/docs/RecPro/CTP.pdf [15 February 2012]

10 Jongkees LB, Maas JP, Philipszoon AJ. Clinical nystagmography. A detailed study of 341 patients with vertigo. Pract Otorhinolaryngol (Basel) 1962;24:65-93

11 Bland M. An Introduction to Medical Statistics, 3rd edn. Oxford: Oxford University Press, 2009

12 Coates AC. The diagnostic significance of spontaneous nystagmus as observed in the electronystagmographic examination. Acta Otolaryngol 1969;67:33-42

13 Department of Health. Provision of Adult Balance Services: A Good Practice Guide. London: Department of Health, 2009

Address for correspondence:

Dr P D B West

Department of Audiovestibular Medicine,

Queen Alexandra Hospital,

Southwick Hill Road,

Cosham,

Portsmouth PO6 3LY, UK

Fax: +44 (0)2392 283238

E-mail: pdbwest@doctors.org.uk

Dr P D B West takes responsibility for the integrity

of the content of the paper

Competing interests: None declared 